Orale Tumore

Ein Ratgeber für Betroffene, Angehörige

und Therapeuten

\section{Sabine Koppetsch}

Idstein, Schulz-Kirchner Verlag, 2006, 68 S., 7,80 EUR

ISBN 3-8248-0344-5

Die Bezeichnung «Ratgeber» ist für meinen Geschmack etwas zu selbstbewusst gewählt und sollte eventuell durch einen Begriff wie z.B. «Informationsbroschüre» ersetzt werden. Meiner Ansicht nach ist die angegebene Zielgruppe (Betroffene und Angehörige) eindeutig zu weit gefasst. Zwar lässt sich nachvollziehen, dass sich die Autorin um eine einfache, auch Laien verständliche Sprache bemüht hat, leider konnte sie dieses Ziel jedoch nur unzureichend erreichen. Mit großer Sicherheit ist der Inhalt auch für vorgebildete Laien deutlich zu anspruchsvoll. Als Zielgruppe für dieses Buch würde ich Therapeuten wie Logopäden, Physiotherapeuten usw. empfehlen, wobei auch diese vermutlich bereits an Grenzen stoßen. Absolut geeignet erscheint mir der «Ratgeber» für pflegerisches Personal aus dem Bereich der Onkologie (z.B. OnkologieFachpflegepersonal).

Teilweise fehlt eine sinnvolle Strukturierung einzelner Anschnitte, welche auch in unlogischer bzw. unglücklicher Reihenfolge auftauchen. Beispielsweise werden auf Seite 17 «Erste Symptome und Prognose» in einem Abschnitt behandelt, was in meinen Augen nicht zusammenpasst. Die Prognose sollte am Ende eines solchen Heftes diskutiert werden. Dann wird auf Seite 20 die «Diagnostik» noch vor den anatomischen Grundlagen (S. 22) aufgeführt und die «Versorgung der Zunge» (hier stellt sich die Frage welche Versorgung?) und die «Zungenmuskeln» (S. 24) anschließend besprochen. Das wirkt meiner Meinung nach eher unprofessionell. Hier sollte zunächst eine logische Gliederung vorgenommen werden.

Das Heft leidet weiterhin unter den kontrastarmen schwarzweiß Bildern. Hierbei sind die anatomischen Strukturen in der Nahaufnahme für den Laien, teilweise auch für Pflegepersonal, nicht zuzuordnen. Farbige Abbildungen wären zeitgemäß und wünschenswert.

Als langjähriger Leiter der hiesigen HNO-onkologischen Tumorstation würde ich diesen «Ratgeber» meinen Patienten und ihren Angehörigen vermutlich nicht an die Hand geben.

A. Sesterhenn, Marburg
Neuro-Oncology of CNS Tumors

J.-C. Tonn, M. Westphal, J.T. Rutka, S.A. Grossmann (Eds.) Heidelberg, Springer, 2006, XVI, 706 S., 260 Abb., 60 Tab., 213,95 EUR

ISBN 3-540-25833-7

«Neuro-Oncology of CNS Tumors», herausgegeben von Tonn, Westphal, Rutka und Grossmann bietet auf etwa 700 Seiten einen umfassenden Überblick über die Tumoren des zentralen und peripheren Nervensystems. Die Herausgeber, hochrangige Neurochirurgen aus Europa, den Vereinigten Staaten und Kanada, haben in ihrer Buchkonzeption ausgewiesene Neurochirurgen und Neuropathologen aus der ganzen Welt zu Wort kommen lassen.

Der Aufbau des Buches ist sehr übersichtlich und gelungen. Es ist in fünf große Teilbereiche gegliedert: Im ersten Teil «Cranial Neuro-Oncology» werden Tumoren im Erwachsenenalter behandelt, im zweiten Teil «Pediatric Neuro-Oncology» Tumoren im Kindesalter, der dritte Teil «Spinal NeuroOncology» stellt die spinalen Tumoren dar, der vierte die peripheren Nervtumoren und im fünften Teil wird auf systemische und generelle Aspekte der Neuro-Onkologie eingegangen. Am Anfang des ersten Teiles steht ein großer und umfassender Beitrag über die Pathologie und Klassifikation der Tumoren. Wesentliche molekulare Erkenntnisse werden dargestellt. Danach folgen in einem gleichartigen Aufbau die einzelnen Kapitel zu den Tumoren, in denen der Epidemiologie, den Symptomen, der Diagnostik, der Therapie, den speziellen Problemen und Komplikationen und den zukünftigen Perspektiven Rechnung getragen wird. Hinweise auf die wichtigsten Literaturstellen runden die jeweiligen Kapitel ab. Die konsequent durchgeführte Gliederung der einzelnen Kapitel gibt einen systematischen Überblick und erleichtert die Aufnahme der Information. Ausgesuchtes Bildmaterial sowie tabellarische Darstellungen unterstreichen den guten Aufbau des Buches. Der Leser erhält damit in anschaulicher und didaktisch hervorragender Weise einen umfassenden Überblick über die einzelnen Tumorentitäten und deren Behandlung.

Ein wichtiger Aspekt des Buches besteht darin, den Unterschied derselben Tumorentität im Kindesalter und Erwachsenenalter hervorzuheben und auf die unterschiedlichen Therapiestrategien einzugehen. Wichtige Syndrome finden Beachtung. Wesentlich für den interessierten Neuro-Onkologen ist die Darstellung der unterschiedlichen Therapiemodalitäten in ihrer Interdisziplinarität. So eignet sich «Neuro-Oncology of CNS Tumors» nicht nur hervorragend für neuroonkologisch interessierte Neurochirurgen, die ihr Wissen über chirurgische

\title{
KARGER \\ (c) 2006 S. Karger GmbH, Freiburg


Strategien und Besonderheiten durch die Diskussion über operative Verfahren und Zugangswege vertiefen können, sondern auch für radioonkologisch tätige Kollegen, Neurologen und internistische Onkologen, die einen Überblick zur Indikation der Strahlentherapie und Chemotherapie erhalten. Die am Ende der jeweiligen Kapitel dargestellte Sektion «Future Perspectives» ist besonders lesenswert, da sie einen interessanten Ausblick in die aktuelle Forschung und künftig mögliche Therapiestrategien bietet.

«Neuro-Oncology of CNS Tumors» bietet eine sehr gelungene Darstellung der Tumoren des zentralen und peripheren Nervensystems im Kindes- und Erwachsenenalter und kann als Lehrbuch, aber auch als interdisziplinäres Nachschlagewerk allen neuroonkologisch tätigen Ärzten uneingeschränkt empfohlen werden. Den Herausgebern und den Autoren der Kapitel darf man zu ihrem Werk gratulieren.

Gabriele Schackert, Dresden

Kurzgefasste interdisziplinäre Leitlinien 2006, 5. Auflage

Deutsche Krebsgesellschaft (Hrsg.)

München, Zuckschwerdt, 2006, 644 S., 29,90 EUR

ISBN 3-88603-891-2

«Kurzgefasst» klingt verheißungsvoll, «interdisziplinär» ist wichtig und «Leitlinien» sind ein Muss - dies ist schon die 5. Auflage dieses Werkes, herausgegeben von einem hochkarätigen Autorenkollektiv im Namen der Deutschen Krebsgesellschaft. In unserer Zeit, in der Information explosionsartig auf uns zukommt und kaum noch verarbeitbar und integrierbar ist, sind solche Richtlinien eine große Hilfe für die tägliche Praxis.

Auf 621 Seiten und in 67 Kapiteln wird versucht, den Stand der Empfehlungen zur Diagnostik und Therapie maligner Erkrankungen darzustellen. Zu Beginn des Buches kommt Prinzipielles wie Chemo-, Strahlen- und Hormontherapie auf den Plan, gefolgt von einem Kapitel über Rehabilitation, ein in Deutschland weit verbreitetes Angebot, welches sich zunehmend den Kriterien der Qualitätssicherung unterzieht. Anschließend geht es von «oben nach unten» und von «innen nach außen», anatomisch gesprochen.
Neu gegenüber der letzten Auflage ist der Part über hämatologische Erkrankungen, kurz und prägnant gehalten, aber all das an molekularbiologischer Erkenntnis der letzten Jahre enthaltend, was einer differenzierten Therapie bedarf, z.B. bei der B-CLL oder beim multiplem Myelom. Die neue WHOKlassifikation der Lymphome wird älteren Klassifikationen gegenübergestellt, auch Hautlymphome werden beschrieben, die ja sonst häufig in hämatologischen Publikationen fehlen.

Den häufigsten Tumorentitäten ist der größte Umfang gewidmet (Mamma- Colonkarzinom, Lungentumoren), wobei sich das Kapitel über das Mammakarzinom etwas holprig liest, da pro Satz zwei bis drei Empfehlungsgrade zitiert werden, hier haben sich die Mitglieder der Organkommission «Mamma» sehr «evidence-based» die Feder führen lassen. Trotzdem oder vielleicht gerade deshalb ist das Kapitel aber sehr umfassend abgehandelt.

In der Mitte des Körpers fehlt plötzlich eine Region - Magen, Pankreas und Leber -, diese Organe werden sicher in der nächsten Auflage berücksichtigt werden. Der Teil über Sarkome ist für den Internisten sehr hilfreich, auch das Kapitel über Kopf- und Halstumoren liefert wertvolle Information über chirurgische und strahlentherapeutische Maßnahmen.

In einigen Kapiteln kommen Dosierungen von Medikamenten vor, während andere hier nicht so konkret sind - an sich wäre eine Sammlung von Dosierungsschemata für die Chemotherapie am Ende des Buches sehr benutzerfreundlich sowie vielleicht ein Stichwortregister.

Der Schwerpunkt «Psychoonkologie» wird im Kapitel der pädiatrischen Onkologie thematisiert, für die Erwachsenen gibt es dazu leider (noch?) kein entsprechendes Kapitel. Sehr praktisch ist der Teil über die Lymphknotenabklärung, die auch gut auf Erwachsene zu übertragen ist. Palliativtherapie und Schmerztherapie sind natürlich unverzichtbar und gut dargestellt, komplementäre Maßnahmen, gerade in Deutschland bei Patienten beliebt, sind kurz angerissen.

Für alle Onkologen die in die «Verlegenheit» kommen, bei Behandlungsentscheidungen über ihre ureigenen Tumorentitäten hinausschauen zu müssen, ist dies ein sehr empfehlenswertes Buch. In der Summe sind die «kurzgefassten interdisziplinären Leitlinien» aber gerade für diejenigen internistischen Onkologen hilfreich, die ein breites diagnostisches und therapeutisches Feld zu versorgen haben.

Johanna Kantner, Innsbruck 